\title{
Elastic 3-D Alignment of Rat Brain Histological Images
}

\author{
Smadar Gefen*, Member, IEEE, Oleh Tretiak, Senior Member, IEEE, and Jonathan Nissanov, Member, IEEE
}

\begin{abstract}
A three-dimensional wavelet-based algorithm for nonlinear registration of an elastic body model of the brain is developed. Surfaces of external and internal anatomic brain structures are used to guide alignment. The deformation field is represented with a multiresolution wavelet expansion and is modeled by the partial differential equations of linear elasticity. A progressive estimation of the registration parameters and the usage of an adaptive distance map reduce algorithm complexity, thereby providing computational flexibility that allows mapping of large, high resolution datasets. The performance of the algorithm was evaluated on rat brains. The wavelet-based registration method yielded a twofold improvement over affine registration.
\end{abstract}

Index Terms-Brain mapping, elastic deformation, neuroinformatics, nonlinear registration, wavelet.

\section{INTRODUCTION}

$\mathbf{T}$ HREE-DIMENSIONAL (3-D) image registration is essential in various computer-vision tasks and as such has been intensively investigated over the past decades. Registration has been applied to many applications including 3-D reconstruction, surveillance operations, efficient image/video representation and retrieval, and medical imaging, albeit with different registration techniques and with different performance requirements. In medicine, image alignment is used to fuse complementary information in order to improve visualization of regions of interest for clinical or research purposes. A survey of various medical image registration techniques can be found in [1]-[3].

A common biomedical problem where image registration has been employed is in mapping newly acquired experimental datasets onto a reference atlas. Registration in this case facilitates automatic segmentation. Alignment of biomedical intersubject images requires a registration algorithm that will compensate as much as possible for geometric variability among individuals. Numerous methods that account for nonlinear differences between subjects have been proposed

Manuscript received June 9, 2003; revised August 25, 2003.This work was supported by the National Institutes of Health (NIH) under Award NS07440. Asterisk indicates corresponding author.

*S. Gefen is with the Computer Vision Laboratory for Vertebrate Brain Mapping, Department of Neurobiology and Anatomy, Drexel University College of Medicine, 2900 Queen Lane, Philadelphia, PA 19129-1096 USA (e-mail: sgefen@drexel.edu).

O. Tretiak is with the Electrical and Computer Engineering Department, Drexel University, Philadelphia, PA 19104 USA.

J. Nissanov is with the Computer Vision Laboratory for Vertebrate Brain Mapping, Department of Neurobiology and Anatomy, Drexel University College of Medicine, Philadelphia, PA 19129-1096 USA (e-mail: nissanov@drexel.edu).

Digital Object Identifier 10.1109/TMI.2003.819280 including modeling one subject as an elastic deformed version of another. For example, Duchon [4] and Meinguet [5] introduced the thin-plate splines (TPS) algorithm as a mathematical interpolator. Later, Goshtasby [6] and Bookstein [7] applied this interpolator to two-dimensional (2-D) image registration. Gabrani and Tretiak [8], [9] extended Bookstein's method to 3-D image registration. Since then many extensions and improvements to Bookstein's TPS-based registration method have been proposed [10]-[12]. Johnson and Chirstensen [10], for instance, have proposed a consistent TPS image registration method that is landmark and intensity based, in which consistency is maintained between forward and backward transformation. Briefly, in the TPS method the image is modeled as a metal plate in which landmark points are deformed in each direction separately. Coefficients of radial basis functions that interpolate the deformation are computed such that an elastic energy functional is minimized.

In contrast to the mathematical thin-plate approach, the Navier linear partial differential equation (PDE) is a physical model of nonlinear elastic deformation. The Navier PDE modeling of organ tissues as an elastic object, adopted from continuum mechanics, considers the organs as elastic media that are exposed to external forces and are smoothly deformed. Bajcsy and Kovacic [13] and Broit [14] were the first to apply Navier PDE to registration. In their method an equilibrium state between external and internal forces was reached for an isotropic homogeneous body. The external forces were determined in such a way that an image-based similarity metric was maximized.

Another approach uses deformable objects, either parametric or level-set-based methods, to track a large nonlinear deformation [15]-[19]. For example, Davatzikos and Prince [17], [18], used a deformable surface parametric representation of the brain's external surface to map one brain surface onto another based on extracted surface (geometric) features. The elastic mapping of one brain volume to another was found by solving the differential equation that models an inhomogeneous elastic body under external forces. The external forces were determined so that the known cortical mapping was satisfied.

Nonlinear registration usually involves finding a minima of a metric that is a function of many registration parameters. Registration failure might occur due to the difficulty in finding a global minimum in the presence of many local minima. The coarse-to-fine strategy in the optimization process can prevent convergence into local minima traps. For example, Thevenaz et al. [20] proposed an image-based linear registration algorithm in which an image pyramid was built using cubic B-spline interpolator and a coarse-to-fine iterative strategy was applied to 
estimate the affine transformation that minimize an intensity difference between a reference and a test data set. Similarly, Shen and Davatzikos [21] have proposed an elastic registration algorithm that is driven by Geometric Moment Invariants (GMI); the GMI features were selected hierarchically and successive approximations of the energy function were minimized. Lester [22] classified nonlinear hierarchical registration methods based on a gradual increase in data complexity, warp complexity, and model complexity.

An alternative representation of the elastic deformation transformation is wavelet multiresolution decomposition. The wavelet, being an inherently hierarchical approximation of a transformation, is naturally suitable for such progressive (coarse-to-fine) optimization starting from the low-resolution approximation of the transformation (global deformation), through the details in its different orientations, and ending with the finest details of the transformation (local deformation).

Wavelets have been used by several researchers for the application of image registration, either in order to represent the nonlinear deformation between two images [23]-[25] or to represent the intensities of images' voxels [26]. For example, Amit [23] used a wavelet basis as well as a Fourier basis to represent the deformation. He used a gradient descent optimization method to minimize the mean of squared distances between intensities of overlapping voxels. Similarly, Downie [24] used a simulated annealing optimization method to find the wavelet coefficients that minimize the sum of squared distances between intensities of corresponding voxels. Both algorithms, [23] and [24], are image-based and as such are most suited for applications dealing with intramodality datasets. In contrast, the registration algorithm developed in this study, is based on geometric features: alignment is guided by samples from the objects' surfaces and, therefore, can be used for applications dealing with intermodality as well as intramodality scans.

In this paper, we are interested in the registration of rat brain images from a cryoplane macroscope. Briefly, with this instrument frozen tissue is cut with a microtome and the revealed blockface surface imaged. The sequential images are in-register by virtue of the accurate repositioning of the tissue underneath the imaging system afforded by our system. Tissue differentiation is achieved by systemic staining, in the present case using black magic ink. As in all histological procedures, intersubject variability includes intensity variations across speciements. Additional deformation is also introduced by the imaging process itself. Solely intensity-based alignment procedure has not proven effective, and so we have adopted geometric-based ones instead. Because as of yet only limited success has been achieved with fully automated segmentation of such geometric features and the high cost in labor associated with manual delineations, we have been motivated to develop a registration method that would rely on only a sparse sample of points on the surface of brain regions.

In a previous study, we presented a 2-D wavelet-based registration method [27]. Here, we extend that method to threedimensions and compare its performance with that of a TPS method [8]. We show how the scalability of the wavelet-based method helps in reducing the complexity introduced by the high resolution 3-D data. We also devised an adaptive distance map to speed up the very time consuming surface distance computations that take place at each iteration of the optimization process. The wavelet-based registration method presented here is guided by sample points from rat brains' external and internal surfaces. A finite-supported, semi-orthogonal wavelet was used to approximate the nonlinear deformation field. The similarity metric used was based on the squared sum of surface distances where a surface distance is defined here by the interval between a point on a test surface and the closest point to it on the corresponding reference surface. The Marquardt-Levenberg (M-L) optimization algorithm is used to minimize a functional that is the sum of two terms, namely the sum of squared distances and the elastic energy.

Often a registration algorithm minimizes a cost function that is a combination of a distance metric and smoothness constraint (regularization) [10], [21], [28]-[31]. There are ample algorithms that iteratively minimize the surface distance in order to linearly align two shapes, sometimes these are referred to as iterative closest point (ICP) algorithms. For example, see [32] for a widely used geometric matching ICP algorithm. In this paper, we are interested in recovering an elastic transformation, therefore, in addition to minimizing the surface distance we minimize the deformation elastic energy. The deformation field between two corresponding objects is recovered progressively, starting with the global part of the deformation and refining with the local deformation components.

This method of surface-based registration that we applied was specially designed for registration of large volume histological data in neurobiology settings. In these setting, it is beneficial to be able to match an experimental (test) brain onto an atlas using only a sparse sample of points from the test brain structure surfaces. In our experiments we used a limited number of samples (less than $1 \%$ of the number of voxels that define each structure's surface) and applied the alignment algorithm to minimize 1) the distance between only these points and the corresponding reference surfaces and 2) the elastic energy. We then evaluated the performance with another larger dataset; we measured the residual distance between this larger validation dataset and the reference brain's surfaces.

The rest of the paper is organized as follows. Section II presents the wavelet representation of the elastic deformation. Next, in Section III, the registration algorithm is described. Sections II and III are an extension of the presentation in Gefen [27] into a 3-D formulation. Alignment experiments, demonstrating the algorithm's performance, are presented in Section IV. We conclude with a discussion in Section V.

\section{ELASTIC DEFormation REPRESENTATION}

A registration algorithm estimates the parameters of a morphological transformation that brings into correspondence a test object and a reference object. When expanding the elastic deformation field $\mathbf{u}(\mathbf{x})$ with a wavelet decomposition, it is necessary to estimate the wavelet parameter vector, $\mathbf{c}$, that yields

$$
\begin{aligned}
& x^{\prime}=x+u^{1}(x, y, z ; \mathbf{c}) \\
& y^{\prime}=y+u^{2}(x, y, z ; \mathbf{c}) \\
& z^{\prime}=z+u^{3}(x, y, z ; \mathbf{c})
\end{aligned}
$$


where $\mathbf{x}=(x, y, z)$ are coordinates in the test object space and $\mathbf{x}^{\prime}=\left(x^{\prime}, y^{\prime}, z^{\prime}\right)$ are the corresponding coordinates in the reference object space. The registration algorithm estimates $\mathbf{c}$ by minimizing 1) the sum of Euclidian distances between points on corresponding surfaces of the test's and the reference's structures and 2) the elastic energy of the deformation.

As mentioned earlier, we use wavelet expansion to approximate the elastic deformation. The 3-D separable wavelet decomposition of a deformation transformation, $\mathbf{u}(\mathbf{x})$, everywhere within a cubical support $\mathbf{N}=\left(N_{x}, N_{y}, N_{z}\right)$ is presented in (1)

$$
\begin{aligned}
u(\mathbf{x})^{i}= & \sum_{\mathbf{k}=0}^{\mathbf{N}_{J}-1} 2^{-J} c_{J \mathbf{k}}^{i 1} \Phi^{1}\left(2^{-J} \mathbf{x}-\mathbf{k}\right) \\
& +\sum_{j=R}^{J} 2^{-j} \sum_{s=2}^{8} \sum_{\mathbf{k}=0}^{\mathbf{N}_{j}-1} c_{j \mathbf{k}}^{i s} \Phi^{s}\left(2^{-j} \mathbf{x}-\mathbf{k}\right) \\
\mathbf{N}_{j} \equiv & 2^{-j} \mathbf{N}=2^{-j}\left(N_{x}, N_{y}, N_{z}\right) \\
\mathbf{k}= & \left(k_{x}, k_{y}, k_{z}\right), \quad \mathbf{x}=(x, y, z), \quad i=1,2,3 .
\end{aligned}
$$

The basis functions are 3-D functions that are translated across a cubical grid with intervals of $2^{j}$ and within a support of $\mathbf{N}=\left(N_{x}, N_{y}, N_{z}\right)$. Each basis function is weighted by the corresponding wavelet coefficient $c_{j \mathbf{k}}^{i s}$. The basis functions are a tensor product of the one-dimensional scaling and wavelet functions as in (2)

$$
\begin{aligned}
& \Phi^{1}=\phi\left(2^{-j} x-k_{x}\right) \phi\left(2^{-j} y-k_{y}\right) \phi\left(2^{-j} z-k_{z}\right) \\
& \Phi^{2}=\phi\left(2^{-j} x-k_{x}\right) \phi\left(2^{-j} y-k_{y}\right) \psi\left(2^{-j} z-k_{z}\right) \\
& \Phi^{3}=\phi\left(2^{-j} x-k_{x}\right) \psi\left(2^{-j} y-k_{y}\right) \phi\left(2^{-j} z-k_{z}\right) \\
& \Phi^{4}=\phi\left(2^{-j} x-k_{x}\right) \psi\left(2^{-j} y-k_{y}\right) \psi\left(2^{-j} z-k_{z}\right) \\
& \Phi^{5}=\psi\left(2^{-j} x-k_{x}\right) \phi\left(2^{-j} y-k_{y}\right) \phi\left(2^{-j} z-k_{z}\right) \\
& \Phi^{6}=\psi\left(2^{-j} x-k_{x}\right) \phi\left(2^{-j} y-k_{y}\right) \psi\left(2^{-j} z-k_{z}\right) \\
& \Phi^{7}=\psi\left(2^{-j} x-k_{x}\right) \psi\left(2^{-j} y-k_{y}\right) \phi\left(2^{-j} z-k_{z}\right) \\
& \Phi^{8}=\psi\left(2^{-j} x-k_{x}\right) \psi\left(2^{-j} y-k_{y}\right) \psi\left(2^{-j} z-k_{z}\right) .
\end{aligned}
$$

The superscript index, " $s$," in (1) marks the subband (orientation) in the wavelet decomposition space.

Multiresolution analysis theory states that a transformation that is approximated by its projection into space $V_{R-1}$ can be equivalently represented by its projection into a lower resolution level space $V_{J}$ and the difference spaces $W_{R}+W_{R+1}+\cdots+W_{J}$ (see Vetterli [33], Strang [34], Mallat [35], and Daubechies [36], for example). The wavelet representation resolution is determined by the application and by the smoothness degree of the transformation.

In general, the number of parameters $c_{j \mathrm{k}}^{i s}$ is the same as the number of grid points in the represented transformation cubical support. For example, in our experiments a support of $\mathbf{N}=$ $\left(N_{x}, N_{y}, N_{z}\right)=(1024,512,512)$ was used. Therefore, 1024 . $512 \cdot 512=268435456$ parameters are required to represent the deformation in one direction. This is a large number of parameters to handle for most minimization algorithms. However, since the transformation of interest - the elastic deformation is by its nature smooth, it can be estimated with only lower resolution levels and still provide reasonable accuracy. For example, the wavelet decomposition of a deformation field with a support of $\mathbf{N}=(1024,512,512)$, and when using the sixth resolution level, contains 1024 parameters. In order to further reduce the number of parameters that the optimization algorithm deals with at a time, the wavelet coefficients can be split into groups that are classified by the orientation and the level of resolution they represent. Each such group of wavelet coefficients corresponds to a different component of the deformation. In other words, the deformation is a combination of components with different levels of details (resolution levels) and orientations (subbands). A progressive approach is applied in which the wavelet coefficients that correspond to a low resolution transformation component are estimated first and the wavelet coefficients that correspond to a high resolution transformation component are estimated last.

\section{Algorithm DeSCRIPTION}

The registration of one elastic object to its homologue is done by estimating the nonlinear deformation that brings together a pair of corresponding surfaces. The deformation is determined by the registration parameters-the wavelet coefficients $c_{j \mathbf{k}}^{i s}$-that minimize the functional $E(\mathbf{c})$. We define a functional $E(\mathbf{c})$ as the weighted sum of 1$)$ the sum of squared surface distances, $e(\mathbf{c})$ and 2) the elastic energy, $L(\mathbf{c})$, so that $E(\mathbf{c})=e(\mathbf{c})+\omega \cdot L(\mathbf{c})$. The parameter $\omega$ is a weight constant that is empirically determined. Sections III-A and B present the derivations of $e(\mathbf{c})$ and $L(\mathbf{c})$ as a function of the registration parameters, respectively. Section III-C describes the progressive approach to the M-L-based optimization procedure applied in the proposed wavelet-based registration algorithm.

\section{A. Sum of Squared Distances e(c)}

The distance between two points, $d_{m} \equiv\left\|d\left(\mathbf{a}\left(\mathbf{x}_{m}, \mathbf{c}\right), S_{r}\right)\right\|$, is the Euclidean distance between an already mapped point on the test surface $\mathbf{a}\left(\mathbf{x}_{m}, \mathbf{c}\right)$ and the closest point to it on the reference surface, $\mathbf{x}_{m}^{\prime} \in S_{\text {ref }}$. Similarly, a vector distance is defined as $\mathbf{v}_{m} \equiv \mathbf{a}\left(\mathbf{x}_{m}, \mathbf{c}\right)-\mathbf{x}_{m}^{\prime}$. Note that $d_{m}=\left\|\mathbf{v}_{m}\right\|$. The magnitude being minimized is the sum of the squared distances

$$
e(\mathbf{c})=\sum_{m=1}^{M} d_{m}^{2}=\sum_{m=1}^{M} d\left(\mathbf{a}\left(\mathbf{x}_{m}, \mathbf{c}\right), S_{r}\right)^{2}
$$

Local dependency of $e(\mathbf{c})$ on the registration parameters, $\mathbf{c}$, is achieved by substituting the Taylor expansion of $d_{m}$ in the vicinity of $\mathbf{c}_{l}$ as follows:

$$
\begin{aligned}
e(\mathbf{c}) \approx & \sum_{m=1}^{M}\left[d_{m}\left(\mathbf{c}_{l}\right)+\Delta^{T} \operatorname{grad}_{c} d_{m}\left(\mathbf{c}_{l}\right)\right]^{2} \\
= & \sum_{m=1}^{M}\left[d_{m}^{2}\left(\mathbf{c}_{l}\right)+2-D_{m}\left(\mathbf{c}_{l}\right) \Delta^{T} \operatorname{grad}_{c} d_{m}\left(\mathbf{c}_{l}\right)+\right. \\
& \left.\Delta^{T} \operatorname{grad}_{c} d_{m}\left(\mathbf{c}_{l}\right) \operatorname{grad}_{c} d_{m}\left(\mathbf{c}_{l}\right) T \Delta\right] \\
= & e\left(\mathbf{c}_{l}\right)+\Delta^{T} \mathbf{b}+\Delta^{T} \mathbf{A} \Delta
\end{aligned}
$$

where

$$
\mathbf{b}_{i} \equiv 2 \sum_{m=1}^{M} d_{m}\left(\mathbf{c}_{l}\right) \frac{\partial d_{m}\left(\mathbf{c}_{l}\right)}{\partial c_{i}}, A_{i j} \equiv 2 \sum_{m=1}^{M} \frac{\partial d_{m}\left(\mathbf{c}_{l}\right)}{\partial c_{i}} \frac{\partial d_{m}\left(\mathbf{c}_{l}\right)}{\partial c_{j}}
$$


and

$$
\Delta \equiv\left(\mathbf{c}-\mathbf{c}_{l}\right) .
$$

Note that in the M-L method only the two first elements of the Taylor expansion of $d_{m}$ are included so that the computation of the Hessian matrix is conveniently avoided [37].

Following the chain rule of differentiation, $\partial d_{m}\left(\mathbf{c}_{l}\right) / \partial c=$ $\operatorname{grad}_{\mathbf{a}} d_{m} \cdot \partial \mathbf{a} / \partial c$. It was shown in [38] that $\operatorname{grad}_{\mathbf{a}} d_{m}=$ $\mathbf{v}_{m} / d_{m}$. In our case, it follows that

$$
\frac{\partial d_{m}\left(\mathbf{c}_{l}\right)}{\partial c}=\frac{\partial d_{m}\left(\mathbf{c}_{l}\right)}{\partial c_{j \mathbf{k}}^{i s}}=\frac{v_{m}^{i}}{d_{m}} \Phi^{s}\left(2^{-j} \mathbf{x}_{m}-\mathbf{k}\right)
$$

\section{B. Elastic Energy $L(c)$}

The second term, $L(\mathbf{c})$, that is being minimized is the elastic energy

$L(\mathbf{c})=$

$\int\left[\mu \sum_{j=1}^{3} \sum_{i=1}^{3}\left(\frac{\partial u^{i}}{\partial x_{j}}\right)^{2}+(\mu+\lambda)\left(\frac{\partial u^{1}}{\partial x_{1}}+\frac{\partial u^{2}}{\partial x_{2}}+\frac{\partial u^{3}}{\partial x_{3}}\right)^{2}\right] d \mathbf{x}$

where $\mu$ and $\lambda$ are the Lame's coefficients that reflect the elastic properties of the medium. In this paper, for simplicity, we chose: $\lambda=0$ and $\mu=1$. Adding spatially varied setting of these coefficients can provide refinement of the elastic model. But doing so is beyond the scope of this paper. Substituting the transformation, $\mathbf{u}$, as represented in (1) results in a linear combination of the integral

$$
h_{p q}^{s t}(j, l, \mathbf{k}, \mathbf{n})=\int \frac{\partial \Phi^{s}\left(2^{-j} \mathbf{X}-\mathbf{k}\right)}{\partial x_{p}} \frac{\partial \Phi^{t}\left(2^{-l} \mathbf{x}-\mathbf{n}\right)}{\partial x_{q}} d \mathbf{x}
$$

where $p, q=1,2$. (An analytic evaluation of this integral is presented in [27].) In order to simplify the expression for the elastic energy, it is assumed that the scaling and wavelet functions used satisfy a principle we called threefold orthogonality [27]. The threefold orthogonality property states that the scaling and wavelet functions are orthogonal to each other and orthogonal to each other's first and second derivatives. The consequence of satisfying this property of threefold orthogonality is that minimizing the elastic energy of the deformation is equivalent to minimizing separately the elastic energy of the different deformation components. In this paper, we merely approximate the threefold orthogonality using a semi-ortogonal wavelet spline of order 3 [27].
Let us continue and develop the expression in (5) considering only the transformation component $u^{i}(j, s)=$ $\sum_{\mathbf{k}=0}^{\mathbf{N}_{j}-\mathbf{1}} c_{j \mathbf{k}}^{i s} \Phi^{s}\left(2^{-j} \mathbf{X}-\mathbf{k}\right)$ for $i-1,2,3$. In this case, (5) is a linear combination of the following terms:

$$
\begin{aligned}
& \int\left[\frac{\partial u^{i}}{\partial x_{p}} \frac{\partial u^{l}}{\partial x_{q}}\right] d \mathbf{x} \\
& =\sum_{\mathbf{n}} \sum_{\mathbf{k}} c_{j k}^{i s} c_{j n}^{l s} \int \frac{\partial \Phi^{s}\left(2^{-j} \mathbf{x}-\mathbf{k}\right)}{\partial x_{p}} \frac{\partial \Phi^{s}\left(2^{-j} \mathbf{x}-\mathbf{n}\right)}{\partial x_{q}} d \mathbf{x} \\
& =\sum_{\mathbf{n}} \sum_{\mathbf{k}} c_{j k}^{i s} c_{j n}^{l s} h_{p q}^{s s}(j, j, \mathbf{k}, \mathbf{n}) \equiv \mathbf{c}_{j}^{i s T} \mathbf{Q}_{j p q}^{s} \mathbf{c}_{j}^{l s} .
\end{aligned}
$$

Accordingly, the energy of the transformation portion that corresponds to level $j$ and orientation $s$ is

$$
\begin{aligned}
L(\mathbf{c})= & 2 \mathbf{c}_{j}^{1 s T} \mathbf{Q}_{j 12}^{s} \mathbf{c}_{j}^{2 s}+2 \mathbf{c}_{j}^{1 s T} \mathbf{Q}_{j 13}^{s} \mathbf{c}_{j}^{3 s}+2 \mathbf{c}_{j}^{2 s T} \mathbf{Q}_{j 23}^{s} \mathbf{c}_{j}^{3 s} \\
& +\mathbf{c}_{j}^{s T}\left(2 \mathbf{Q}_{j 11}^{s}+\mathbf{Q}_{j 22}^{s}+\mathbf{Q}_{j 33}^{s}\right) \mathbf{c}_{j}^{1 s} \\
& +\mathbf{c}_{j}^{2 s T}\left(\mathbf{Q}_{j 11}^{s}+2 \mathbf{Q}_{j 22}^{s}+\mathbf{Q}_{j 33}^{s}\right) \mathbf{c}_{j}^{2 s} \\
& +\mathbf{c}_{j}^{3 s T}\left(\mathbf{Q}_{j 11}^{s}+\mathbf{Q}_{j 22}^{s}+2 \mathbf{Q}_{j 33}^{s}\right) \mathbf{c}_{j}^{3 s} .
\end{aligned}
$$

The matrix form of (8) is

$$
L(\mathbf{c})=\mathbf{c}^{T} \mathbf{Q}_{j}^{s} \mathbf{c}
$$

where the equation shown at the bottom of the page holds. We proceed with applying the Taylor expansion of $L(\boldsymbol{c})$ in the vicinity of $c_{l}$ to (9)

$$
\begin{aligned}
L(\mathbf{c}) & \cong \mathbf{c}_{l}^{T} \mathbf{Q}_{j}^{s} \mathbf{c}_{l}+\left(\mathbf{c}-\mathbf{c}_{l}\right)^{T} 2 \mathbf{Q}_{j}^{s} \mathbf{c}_{l}+\left(\mathbf{c}-\mathbf{c}_{l}\right)^{T} \mathbf{Q}_{j}^{s}\left(\mathbf{c}-\mathbf{c}_{l}\right) \\
& =\mathbf{c}_{l}^{T} \mathbf{Q}_{j}^{s} \mathbf{c}_{l}+\Delta^{T} 2 \mathbf{Q}_{j}^{s} \mathbf{c}_{l}+\Delta^{T} \mathbf{Q}_{j}^{s} \Delta .
\end{aligned}
$$

It can be shown that the matrix $\mathbf{Q}_{j}^{s}$ is linearly proportional to the Wavelet-Galerkin discretization matrix of the homogenous static Navier PDE [27]. This implies that minimizing the elastic energy is equivalent to solving the Navier PDE.

The dimension of the matrix $\mathbf{Q}_{j}^{s}$ is $\left[3\left(2^{-j} N_{x} \cdot 2^{-j} N_{y}\right.\right.$. $\left.\left.2^{-j} N_{z}\right), 3\left(2^{-j} N_{x} \cdot 2^{-j} N_{y} \cdot 2^{-j} N_{z}\right)\right]$. Hence, in the higher levels of resolution, due to the increased wavelet basis functions' locality, the matrix $\mathbf{Q}_{j}^{s}$ is large. Note though that the matrix $\mathbf{Q}_{j}^{s}$ depends only on the chosen support, $\mathbf{N}$, and the scaling and wavelet basis functions $\Phi_{j}^{s}$. $\mathbf{Q}_{j}^{s}$ does not depend on the object of interest (more specifically the objects' shape and relative deformation). Therefore, $\mathbf{Q}_{j}^{s}$ can be computed once offline and reused thereafter for the deformation estimation of any input object data.

and

$$
\mathbf{Q}_{j}^{s} \equiv\left[\begin{array}{ccc}
2 \mathbf{Q}_{j 11}^{s}+\mathbf{Q}_{j 22}^{s}+\mathbf{Q}_{j 33}^{s} & \mathbf{Q}_{j 12}^{s} & \mathbf{Q}_{j 13}^{s} \\
\mathbf{Q}_{j 21}^{s} & \mathbf{Q}_{j 11}^{s}+2 \mathbf{Q}_{j 22}^{s}+\mathbf{Q}_{j 33}^{s} & \mathbf{Q}_{j 23}^{s} \\
\mathbf{Q}_{j 31}^{s} & \mathbf{Q}_{j 32}^{s} & \mathbf{Q}_{j 11}^{s}+\mathbf{Q}_{j 22}^{s}+2 \mathbf{Q}_{j 33}^{s}
\end{array}\right]
$$

$$
\mathbf{c} \equiv\left[\begin{array}{lll}
\mathbf{c}_{j}^{1 s T} & \mathbf{c}_{j}^{2 s T} & \mathbf{c}_{j}^{3 s T}
\end{array}\right]^{T}
$$




\section{Parameters Optimization}

The expressions developed above for the sum of the squared distances, $e(\mathbf{c})$, and for the elastic energy, $L(\mathbf{c})$, are combined to result in the functional $E(\mathbf{c})$

$$
\begin{aligned}
E(\mathbf{c}) & =e(\mathbf{c})+\omega \cdot L(\mathbf{c}) \\
& \approx e\left(\mathbf{c}_{l}\right)+\mathbf{c}_{l}^{T} \mathbf{Q} \mathbf{c}_{l}+\Delta^{T}\left(\mathbf{b}+2 \omega \mathbf{Q} \mathbf{c}_{l}\right)+\frac{1}{2} \Delta^{T}(\mathbf{A}+2 \omega \mathbf{Q}) \Delta .
\end{aligned}
$$

Based on our experiments, the parameter $\omega$ was set arbitrarily to 1 . The overall minimization algorithm had as an input a subset of samples from surfaces of the test brain's structures. Accordingly, a range of wavelet resolution can be used for the alignment. The estimated wavelet coefficients included only those that corresponded to wavelet basis functions whose support overlapped the surfaces of the test brain's structures. Starting with zero for coefficient values, an iterative procedure was used to compute coefficients that minimized the functional defined in (11). Iterations were within resolution levels and orientation, using the M-L optimization algorithm [39]. Each iteration involved computing the locations of the deformed test brain, computing surface distances (the distance between a point on the surface of the test brain's structure and the closest point to it on the surface of the corresponding reference brain's structure), and evaluating matrix $\mathbf{A}$ and vector $\mathbf{b}$.

Using a coarse-to-fine approach, we first solved for the coefficients $\hat{\mathbf{c}}_{J}^{1}$, considering only the lowest resolution level where $j=J$ and $s=1$. Next, we proceeded to the second subband, $s=2$ (still at the same resolution level, $j=J$ ), solving for $\hat{\mathbf{c}}_{J}^{2}$. In the same manner, we solved for the other six subbands, $s=3$ through $s=8$, at resolution level $j=J$. We proceeded down the pyramid to the next level of resolution $j=J-1$, solving for the different subbands at this level, $s=2, \ldots, 8$. Hence, the computation of the wavelet coefficients was done progressively until the estimation of all coefficients was completed. Note that since threefold orthogonality is not satisfied (merely semi-orthogonal wavelet was used), the order in which the subbands are chosen plays a role. Indeed different orders will result in slightly different results. This can be dealt with by iterating several times through the different subbands within a specific resolution level. Our experiments showed that more than one iteration yields only slight improvement in accuracy.

\section{EXPERIMENTS}

In this section, the performance and computational advantages of the wavelet-based method are demonstrated. Seven rat brains were reconstructed from cryoplane macroscope images to yield $40 \mu \mathrm{m}$ isotropic 3-D datasets. One of these was defined as our reference atlas while the rest were used as test brains. The data used to drive the alignment algorithm consisted of manually delineated surfaces of external and internal structures of the brain (see Table I). The shape of these structures and their relative position in the brain are shown in Fig. 1.

Manual delineation of surfaces of a brain's anatomical structures is tedious and time consuming, and full delineation is sometimes impossible due to a local lack of contrast caused by a nonuniform distribution of the systemically applied stain.
TABLE I

The Cube Dimension That Inscribes EACH Brain STRUCTURE AND THE VOXELS Number THAT REPRESENT EACH BRAIN STRUCTURE's SURFACE

\begin{tabular}{l|c|c|c|c}
\hline & \multicolumn{3}{|c|}{ Cube dimension } & Voxels \\
\cline { 1 - 3 } & $\mathrm{X}$ & $\mathrm{Y}$ & $\mathrm{Z}$ & number \\
\hline External Surface & 561 & 398 & 411 & 711716 \\
\hline V34 - third and forth ventricle & 154 & 122 & 181 & 35070 \\
\hline MCP - middle cerebellar peduncle & 130 & 152 & 72 & 8142 \\
\hline LV - lateral ventricle & 161 & 202 & 66 & 18998 \\
\hline TPZ - trapezoid & 134 & 147 & 62 & 16809 \\
\hline MC - midline of cerebrum & 432 & 243 & 287 & 50136 \\
\hline N7 - seventh nerve & 79 & 114 & 57 & 9854 \\
\hline OCT - optic chiasm and tracks & 86 & 129 & 76 & 13105 \\
\hline TG5 - trigeminal nerve & 181 & 186 & 110 & 36692 \\
\hline CC - corpus callosum & 174 & 214 & 174 & 57444 \\
\hline CT - cerebellar tracks & 163 & 220 & 172 & 114344 \\
\hline CC+MC - corpus callosum- & 32 & 17 & 44 & 4665 \\
midline intersection & & & & \\
\hline SC - superior colliculus & 39 & 33 & 31 & 5912 \\
\hline AC - anterior commissure & 213 & 206 & 69 & 49895 \\
\hline CP - cerebral peduncle & 57 & 52 & 1 & 3938 \\
\hline
\end{tabular}

Therefore, the alignment of experimental data (test brains) onto an atlas (reference brain) when based only on a small subset of sample points from the surfaces of a test brain's structures can be of great value. Table I shows the dimension of the cube that inscribes each of the brain structures employed by this study as well as the number of voxels needed to fully represent their surface.

In the following experiments, we sampled surface points at a distance of about 30 voxels from each other (less than $1 \%$ of the samples that were needed to represent the surface of the external and internal structures of the test brain). We used this small subset to guide the registration. We validated the registration accuracy based on another larger subset of points. In a simulated case where the true correspondences are known, the true error can be computed as the average Euclidian distances between known corresponding points. In a real case, however, where the true correspondences are unknown, only the surface error can be computed. Surface error is defined here as the average Euclidian distance, where a distance is measured between a point on the surface of a test brain's structure and the closest point to it on the corresponding reference brain's structure.

\section{A. Recovering Synthetic Deformation}

The efficacy of the wavelet-based method to align one rat brain volume to another rat brain volume was assessed first using one brain volume. The evaluation involved three steps: 1) applying a known deformation to the test brain to generate the reference brain; 2) registering the test brain onto the reference brain; and 3) comparing the results of the estimated deformation with the known deformation.

Prior to registration, the test brain was positioned at the center of a $1024 \times 512 \times 512$ cube. Polynomial mapping

$$
\sum_{0 \leq(i+j+k) \leq 4} \mathbf{a}_{i j k} x_{1}^{i} \cdot x_{2}^{j} \cdot x_{3}^{k}
$$

was applied to the test brain to create the reference brain. Fig. 2 shows the test brain with the absolute deformation between reference and test brains coded in gray scale on its surface. Table II 


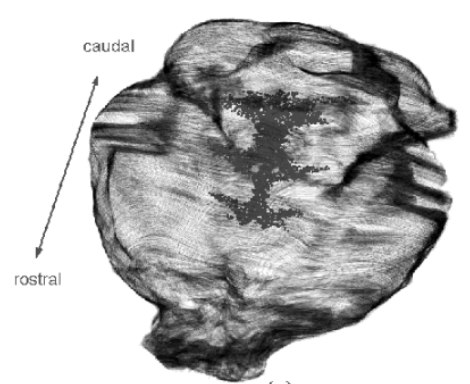

(a)

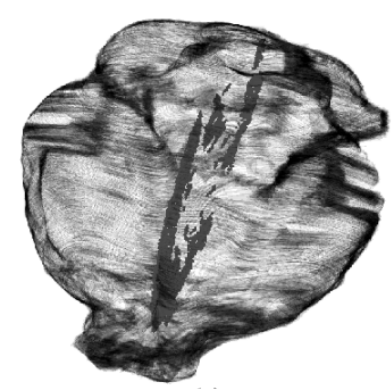

(e)

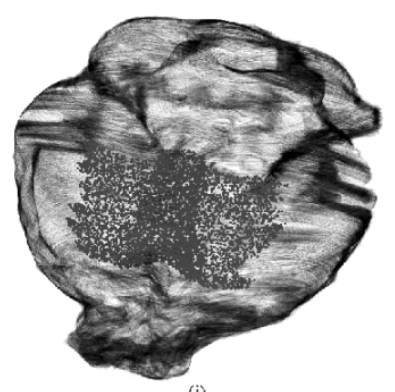

(i)

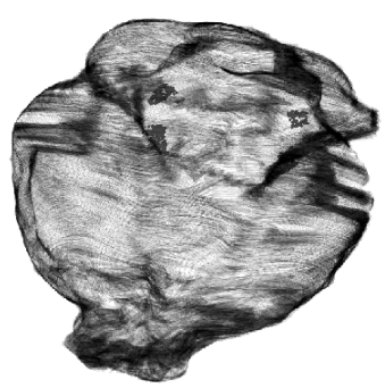

(b)

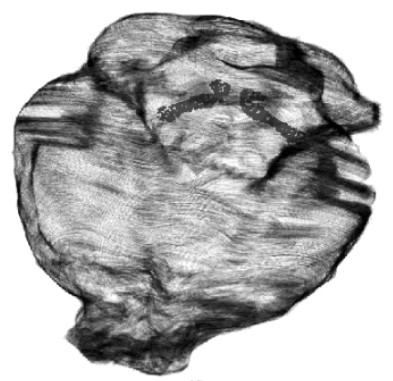

(f)

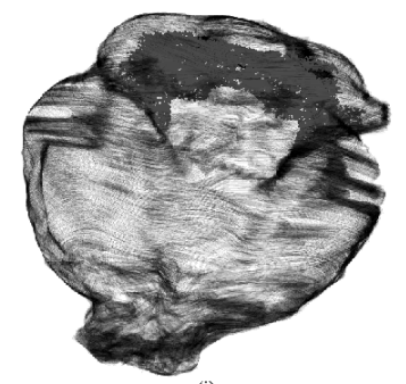

(j)

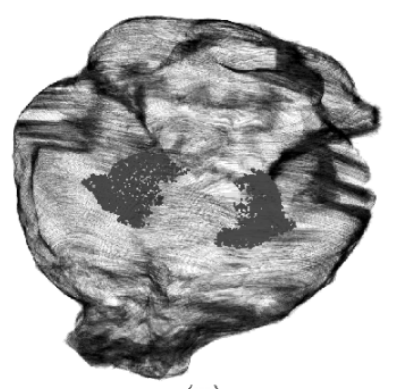

(m)

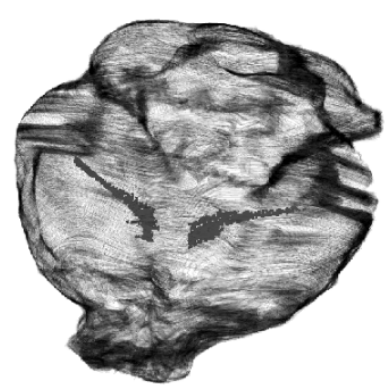

(c)

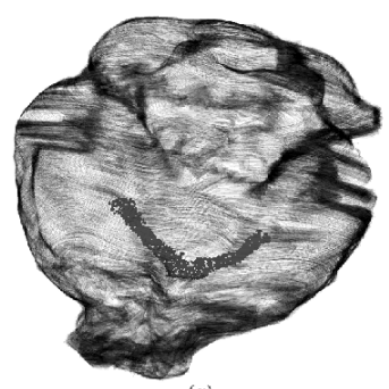

(g)

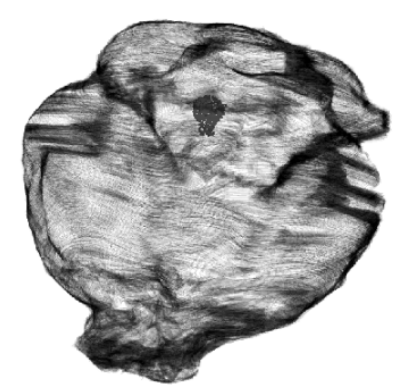

(k)

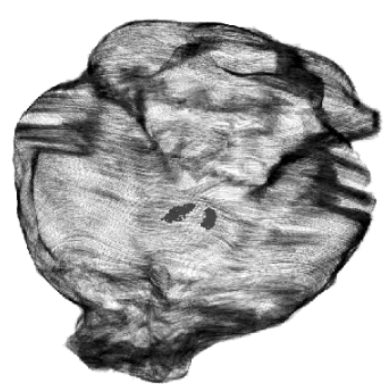

(n)

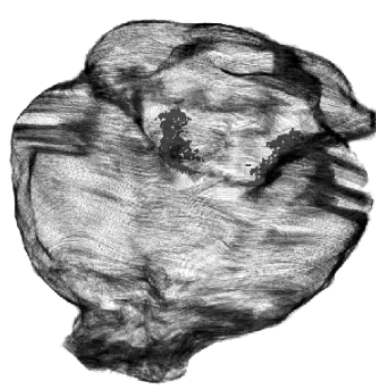

(d)

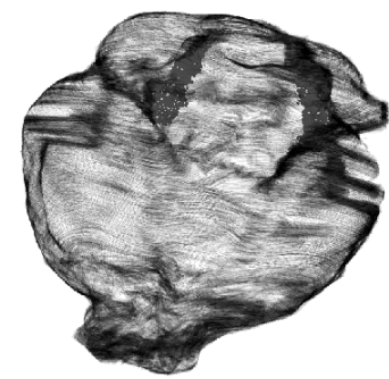

(h)

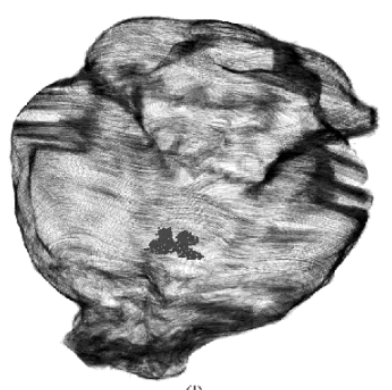

(I)

Fig. 1. Volumes of interest employed to asses algorithm performance. Shown is a ventral view with the external surface rendered semi-opaque. Internal structures are shown in darker opaque shade: (a) Third and forth ventricle, (b) middle cerebellar peduncle, (c) lateral ventricle, (d) trapezoid, (e) midline of cerebrum, (f) seventh nerve, (g) optic chiasm and tracks, (h) trigeminal nerve, (i) corpus callosum, (j) cerebellar tracks, (k) corpus callosum-midline intersection, (l) superior colliculus, (m) anterior commissure, and (n) cerebral peduncle.

shows the mean and median of the applied deformation on the surfaces of the external and internal structures. This deformation was represented by a semi-orthogonal spline wavelet (of order 3) where $J=8$ and $R=6$.

Two cases were examined: 1) when the optimization algorithm used the known correspondences to compute the distance between the test's sample points and the reference (known CPs); and 2) when the optimization algorithm used surface distance to compute the distance between the test's sample points and the reference (unknown CPs). In either case, performance was assessed computing the true error based on the known CPs. The means and the medians of the alignment errors for the different structures are shown in Table II. In the known CPs case, the average error was reduced from about 9 voxels to 0.3 voxels while in the unknown CPs case the error was reduced from about 9 voxels to 0.8 voxels. These results demonstrate that the better the surface distance estimates the distance between true corresponding points the better the registration accuracy. In other words, the average error for the first case can be considered 


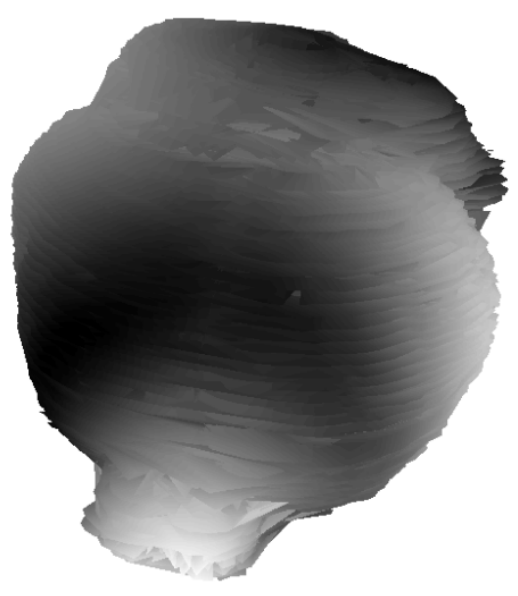

(a)

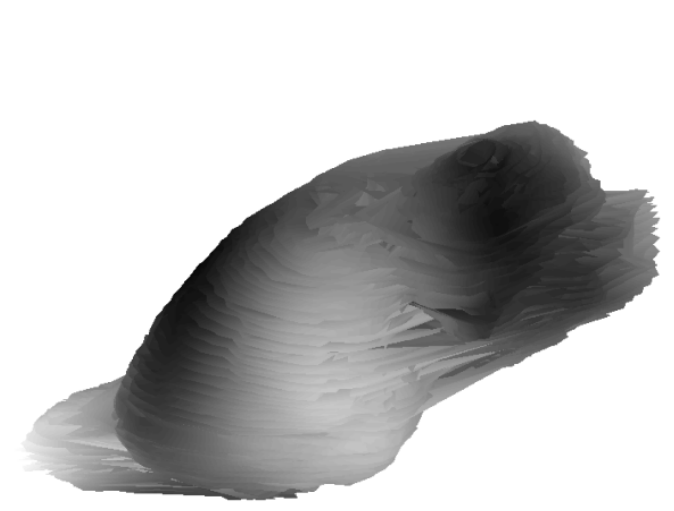

(b)

Fig. 2. The test brain with the applied polynomial deformation magnitude coded on its surface, and shown at (a) horizontal rotation of -37.5 and (b) $37.5^{\circ}$ and vertical elevation of $30^{\circ}$. Values on the gray scaled bar shown on the right are in voxels.

as a lower bound of the algorithm performance in the setting of synthetic deformation.

\section{B. Adaptive Distance Map}

During registration, one of the operations that contributes significantly to algorithm complexity, in terms of both storage and time, is the computation of surface distances. A surface distance value needs to be computed for each sample point on the surface of the test brain's structures; this computation takes place on each iteration of the optimization algorithm, as described in the previous section. As mentioned earlier, the surface distance is the distance between a point on the surface of a test brain's structure and the closest point to it on the surface of the corresponding reference brain's structure. For example, if the surface distance associated with a point on the external surface of the test brain is needed, a search for the closest point to it among 711716 reference points is necessary (see Table I).
TABLE II

WAVELET-BASED REGISTRATION ERROR (IN VOXELS)

\begin{tabular}{l|c|c|c|c|c|c}
\hline \multirow{2}{*}{ surfaces } & \multicolumn{2}{|c|}{$\begin{array}{c}\text { Initial } \\
\text { deformation }\end{array}$} & \multicolumn{4}{c}{ True Error } \\
\cline { 2 - 7 } & mean & median & mean & median & mean & median \\
\hline External & 9.76 & 9.75 & 0.34 & 0.30 & 2.24 & 1.95 \\
\hline V34 & 7.90 & 7.68 & 0.32 & 0.27 & 0.73 & 0.58 \\
\hline MCP & 10.18 & 10.35 & 0.19 & 0.19 & 0.46 & 0.46 \\
\hline LV & 7.36 & 7.30 & 0.30 & 0.23 & 0.45 & 0.22 \\
\hline TPZ & 10.38 & 10.57 & 0.17 & 0.17 & 0.79 & 0.56 \\
\hline MC & 8.62 & 8.36 & 0.29 & 0.23 & 0.83 & 0.69 \\
\hline N7 & 9.85 & 9.68 & 0.57 & 0.59 & 0.76 & 0.88 \\
\hline OCT & 9.13 & 9.19 & 0.18 & 0.17 & 0.96 & 0.77 \\
\hline TG5 & 9.82 & 10.57 & 0.23 & 0.20 & 0.87 & 0.55 \\
\hline CC & 7.72 & 7.58 & 0.31 & 0.27 & 0.69 & 0.48 \\
\hline CT & 9.10 & 8.96 & 0.41 & 0.41 & 0.64 & 0.54 \\
\hline CC+MC & 8.97 & 9.01 & 0.13 & 0.12 & 0.27 & 0.22 \\
\hline SC & 7.27 & 7.24 & 0.23 & 0.23 & 0.38 & 0.35 \\
\hline AC & 8.50 & 8.53 & 0.37 & 0.31 & 1.03 & 1.01 \\
\hline CP & 7.95 & 7.87 & 0.47 & 0.45 & 1.11 & 1.07 \\
\hline Average & $\mathbf{8 . 8 3}$ & $\mathbf{8 . 8 4}$ & $\mathbf{0 . 3 0}$ & $\mathbf{0 . 2 8}$ & $\mathbf{0 . 8 1}$ & $\mathbf{0 . 6 9}$ \\
\hline & & & \multicolumn{3}{c}{} &
\end{tabular}

One approach to speed-up the surface distance computation is to use a discrete distance map. In this approach, surface distances between points on a cubical grid and a reference structure are precalculated and stored in a 3-D array-referred to as a distance map [38]. Later, the registration algorithm accesses this distance map to extract a desired surface distance. Although the usage of a distance map does speed the surface distance computation enormously, it has two drawbacks. First, only distance values associated with grid points can be extracted from the distance map, meaning that the surface distance value at an arbitrary point is not available. (One way to deal with this is to estimate the required distance using a linear interpolation (LI) of the eight neighboring grid points' distance values.) Second, the distance map requires a lot of memory since it needs to contain the distance values of cubical grid points with support that should at least inscribe the union of the test and reference brains. A typical size of the distance map of the reference brain's external surface is $592 \times 432 \times 448$. Assuming that each distance is stored in 3 bytes, the total memory required for this distance map is about 328 Mbytes. Note that a separate distance map should be precalculated for each of the fourteen internal structures of the reference brain as well.

The above approach, although leading to efficiency in computation time, is inefficient in the following aspect. Since it is not known a head of time at what locations distance values will be required by the algorithm, the distances for grid points everywhere within the cubical support needs to be precalculated and stored. This results in a large storage of unused distance values since usually computation of distance is required for points at the vicinity of the test brain's surfaces as it progress toward the corresponding reference brain's surfaces.

In this paper, we reduced the memory size required to store the distance map applying an on-demand construction of the distance map. Instead of populating all of the distance map prior to registration, we initialized an "empty" distance map. During registration when a distance value at a certain point was required an inquiry was done to the distance map to check if this distance 
TABLE III

The AdAPTIVE Distance MAP MACRO-VOXEl MEMORY AllocATION AND SuRfACE DisTANCE COMPUTATION ACCURACY

\begin{tabular}{l|c|c|c}
\hline & $\begin{array}{c}\text { Maximum } \\
\text { MV no. }\end{array}$ & $\begin{array}{c}\text { Allocated } \\
\text { MV no. }\end{array}$ & $\begin{array}{c}\text { Distance est. } \\
\text { error [voxels] }\end{array}$ \\
\hline Ext. Surface & 27972 & 2910 & 0.16 \\
\hline V34 & 1716 & 133 & 0.20 \\
\hline MCP & 960 & 41 & 0.12 \\
\hline LV & 1248 & 69 & 0.15 \\
\hline TPZ & 726 & 61 & 0.17 \\
\hline MC & 10353 & 297 & 0.09 \\
\hline N7 & 528 & 46 & 0.20 \\
\hline OCT & 616 & 53 & 0.14 \\
\hline TG5 & 1890 & 141 & 0.21 \\
\hline CC & 2912 & 269 & 0.27 \\
\hline CT & 2873 & 385 & 0.26 \\
\hline CC+MC & 80 & 21 & 0.12 \\
\hline SC & 100 & 21 & 0.22 \\
\hline AC & 1440 & 198 & 0.25 \\
\hline CP & 126 & 14 & 0.11 \\
\hline Average & $\mathbf{3 5 6 9}$ & $\mathbf{3 1 1}$ & $\mathbf{0 . 1 8}$ \\
\hline
\end{tabular}

value has already been computed and stored by a previous inquiry. If the distance value did not exist in the distance map, it was computed and stored for future inquiries.

The memory allocation and access were done as follows. The distance map volume was partitioned into macro-voxels of $16^{3}$ voxels. A macro voxel was allocated only when a distance value at a point located within its support was required. A distance value at a certain point is accessed by a pointer to the corresponding macro-voxel and another pointer to its location within the macro-voxels. Each time a distance value was required, one of the following scenarios took place.

1) If the macro-voxel associated with this point was allocated and contained all the necessary data, then the interpolated distance value was returned.

2) If the macro-voxel associated with this point was allocated but some or all of the distance values at eight neighboring grid points were not stored in the buffer, then the missing distance values were computed and stored in the macro-voxel buffer and the required distance value was interpolated.

3) If the macro-voxel associated with this point was not allocated, then a macro-voxel buffer was allocated, the distance values of eight neighboring grid points were computed and stored in the macro-voxel buffer, and the required distance value was interpolated.

This adaptive on-demand construction of the distance map resulted in a significant reduction in the memory size needed to accommodate the distance map. Table III shows the maximum number of macro-voxels versus the number of actually allocated ones for each of the brain structures' distance maps. It can be seen that the adaptive distance map required on average ten times less memory than a full precalculated distance map. Table III also shows the estimation error that is the result of using LI for the surface distance computation. Experiments with registration of synthetically deformed test brain showed that the algorithm's running time when using an exact surface distance computation (no distance map is used) is $\sim 120$
TABLE IV

AVERAGE Surface ERROR FOR AFFINE AND WAVELET-BASED REGISTRATION

\begin{tabular}{l|c|c|c|c}
\hline & \multicolumn{2}{|c|}{ Affine } & \multicolumn{2}{c}{ Wavelet } \\
\hline & mean & median & mean & median \\
\hline Ext. Surface & 4.54 & 3.27 & 2.11 & 1.41 \\
\hline V34 & 7.56 & 4.62 & 2.94 & 1.56 \\
\hline MCP & 9.06 & 8.13 & 4.28 & 3.08 \\
\hline LV & 5.07 & 3.90 & 2.20 & 1.15 \\
\hline TPZ & 5.55 & 4.51 & 2.09 & 1.36 \\
\hline MC & 2.37 & 1.83 & 1.20 & 0.83 \\
\hline N7 & 6.99 & 6.57 & 2.15 & 1.76 \\
\hline OCT & 5.86 & 3.88 & 2.77 & 2.17 \\
\hline TG5 & 5.39 & 3.08 & 2.30 & 1.69 \\
\hline CC & 2.57 & 2.23 & 1.42 & 1.10 \\
\hline CT & 5.60 & 3.94 & 2.79 & 1.84 \\
\hline CC+MC & 4.84 & 4.88 & 2.47 & 1.56 \\
\hline SC & 3.61 & 3.25 & 2.30 & 1.62 \\
\hline AC & 2.37 & 1.77 & 2.18 & 1.55 \\
\hline CP & 5.07 & 4.06 & 2.62 & 1.82 \\
\hline Average & 5.10 & 3.99 & 2.39 & 1.63 \\
\hline
\end{tabular}

TABLE V

SURFACE ERRORS (IN VOXELS) AS A FUNCTION OF THE RESOLUTION LEVELS

\begin{tabular}{c|l|l|c|c}
\hline Res. & \multicolumn{2}{|c|}{ Number of Parameters } & \multicolumn{2}{c}{ Surface Error } \\
\hline J-R & Maximum & Actual & mean & median \\
\hline $8-8$ & $\mathbf{1 6 \times 8}$ & $\sim \mathbf{1 6 \times 8}$ & 3.43 & 2.41 \\
\hline $8-7$ & $\mathbf{1 6} 8+\mathbf{1 2 8} \times 7$ & $\sim \mathbf{1 6 \times 8 + 8 5 \times 7}$ & 2.85 & 2.02 \\
\hline $8-6$ & $\mathbf{1 6} 8+\mathbf{1 2 8} \times 7+\mathbf{1 0 2 4} \times 7$ & $\sim \mathbf{1 6} \times 8+85 \times 7+\mathbf{3 0 0} \times 7$ & 2.39 & 1.63 \\
\hline
\end{tabular}

min and when using LI (adaptive distance map is used) is $\sim 17$ min. Hence, using an adaptive distance map the algorithm runs about seven times faster (based on algorithm implementation using MATLAB on a Pentium IV PC with $1.9 \mathrm{GHz}$ and $2 \mathrm{~GB}$ RAM). Interpolation error though is on average 0.18 pixels (see Table III).

\section{Rat Brains Mapping}

To evaluate the algorithm in the intersubject setting, we registered the six rat test brains onto the reference brain. It should be noted that in this case only the surface error was measured since the true error is unknown. Prior to the wavelet-based registration the brains were linearly aligned using a surface-based affine algorithm [40]. Table IV shows the average registration errors of the six different test brains. The errors were computed both after affine alignment and after wavelet-based alignment. As can be seen from Table IV, the average error was reduced from 5.1 to 2.4 voxels.

The progressive refinement of the deformation estimation is demonstrated in Table $\mathrm{V}$. Table $\mathrm{V}$ shows the registration error for the rat brain surface alignment as a function of an increased resolution level. The maximum wavelet coefficients that are contained in a support of $1024 \times 512 \times 512$ are shown beside the actual number of wavelet coefficients that were used. The actual number of wavelet coefficients used corresponds to the wavelet basis functions that overlap the test brain's structures. Only these coefficients were estimated. This procedure resulted in a considerable reduction in complexity. Another reduction in complexity was achieved by considering only lower resolution level of the wavelet representation, where $J=8$ and $R=6$. 
TABLE VI

SURFACE ERRoRS (IN VOXELS) FOR THE WAVELET-BASED AND THE TPS REGISTRATION METHODS

\begin{tabular}{c|c|c|c|c|c|c|c}
\hline \multicolumn{4}{c|}{ Wavelet-based Method } & \multicolumn{4}{c}{ Thin-plate Spline } \\
\hline $\begin{array}{c}\text { Param. } \\
\text { No. }\end{array}$ & mean & median & $\begin{array}{c}\text { Time } \\
{[\mathrm{sec}]}\end{array}$ & $\begin{array}{c}\text { Param. } \\
\text { No. }\end{array}$ & mean & median & $\begin{array}{c}\text { Time } \\
{[\mathrm{sec}]}\end{array}$ \\
\hline$\sim 128$ & 3.43 & 2.41 & 450 & $\sim 128$ & 4.10 & 2.89 & 11 \\
\hline$\sim 700$ & 2.85 & 2.02 & 600 & $\sim 700$ & 3.04 & 1.99 & 75 \\
\hline$\sim 2800$ & 2.39 & 1.63 & 715 & & & & \\
\hline
\end{tabular}

Finally, a comparison between the performance of wavelet-based and the TPS [8] algorithms was done. As is described in Gabrani [8] the TPS interpolates the elastic deformation between two objects using radial functions. Briefly, the TPS method provides a closed form solution in the form of a linear system that satisfies a known deformation at a given number of corresponding points. In this method, the number of registration parameters is directly proportional to the number of corresponding points used. Hence, the dimension of the linear system is threefold the number of corresponding points. Consequently, solving this system becomes more difficult as the number of parameters grows. Moreover, the linear system is not sparse, so methods that take advantage of sparsity cannot be used.

In our experiments with the TPS method, we estimated the required corresponding points using a surface distance. Table VI shows the average errors for the TPS and the wavelet-based methods when using different numbers of registration parameters. Unlike the wavelet-based method, the TPS cannot resolve the registration parameters in a progressive manner and is, therefore, limited in the number of parameters it can handle. It can be seen, in Table VI, that the wavelet-based method, because it can deal with more estimation parameters, can potentially result in a better alignment when higher resolution levels are used. On the other hand, the TSP, being a closed-form method, takes much shorter time to run.

\section{CONCLUSION}

The nonlinear morphological difference between two corresponding organs complicates matching their features. Alignment of brain histological images further adds to this complexity because of the need to process high resolution data. Hence, a registration algorithm suitable to the application of histological brain mapping should have computational flexibility and scalability so that a highly nonlinear matching of large images will be feasible.

In this paper, a registration algorithm was evaluated that offers a trade-off between complexity and accuracy. This algorithm recovers the deformation field between two brains in a progressive manner: global deformation is restored first following a refinement that is produced by estimating additive deformation details. This hierarchical approach is enabled by a wavelet multiresolution representation of the deformation field. A M-L optimization algorithm is used to find the wavelet coefficients that minimize the distance between corresponding surfaces of brain structures. To maintain the smoothness of the deformation also the elastic energy is minimized. Problem for- mulation results in sparse matrices and decoupling of equations that correspond to different orientations and resolution levels.

The alignment method proposed here is a surface-based multidimensional registration algorithm that is guided by a small number of sample points from the surfaces of a test brain structures. The average error results, obtained from the registration of six rat brains, show a more than twofold improvement over affine alignment. The scalability of the wavelet-based algorithm was demonstrated and was shown to be a possible attractive alternative especially when compared with the TPS. The TPS method, although it provides a closed form solution, is limited in its numerical capability to handle a large number of registration parameters. In addition, to get accurate results the TPS method requires as an input a large number of corresponding points that do not always exist when dealing with anatomical surfaces.

In our experiments, we found that the algorithm's accuracy depends on how well the surface distance estimate the true distance between corresponding points (see Table II). We believe that using contrast information in the vicinity of the surfaces of corresponding brains' structures will lead to a more accurate estimate of the distance between corresponding points. In the future we plan to explore a hybrid approach in which voxel-based features, besides geometric features, will be used to guide the registration.

\section{REFERENCES}

[1] I. N. Bankman, Handbook of Medical Imaging. San Diego, CA: Academic, 2000.

[2] A. W. Toga, Brain Warping. New York: Academic, 1999.

[3] J. B. A. Maintz and M. A. Viergever, "A survey of medical image registration," Med. Image Anal., vol. 2, pp. 1-36, 1998.

[4] J. Duchon, "Interpolation des functions de deux variables suivant le principle de la flexion des plaques minces," in RAIRO Analyze Numerique, vol. 10, 1976, pp. 5-12.

[5] J. Meinguet, "Multivariate interpolation at arbitrary points made simple," Z. Angew. Math. Phys., vol. 30, pp. 292-304, 1979.

[6] A. Goshtasby, "Registration of images with geometric distortions," IEEE Trans. Geosci. Remote Sensing, vol. 26, pp. 60-64, Jan. 1988.

[7] F. L. Bookstein, "Principal warps: Thin-plate splines and the decomposition of deformations," IEEE Trans. Pattern Anal. Machine Intell., vol. 11, pp. 567-585, June 1989.

[8] M. Gabrani, "Multidimensional spline theory and surface-based alignment of brains," Ph.D. dissertation, Elect. Comput. Eng., Drexel Univ., Philadelphia, PA, 1998.

[9] M. Gabrani and O. J. Tretiak, "Surface-based matching using elastic transformations," Pattern Recogn., vol. 32, pp. 87-97, 1999.

[10] H. J. Johnson and G. E. Christensen, "Consistent landmark and intensity-based image registration," IEEE Trans. Med. Imag., vol. 21, pp. 450-461, May 2002.

[11] J. F. Krucker, G. L. LeCarpentier, J. B. Fowlkes, and P. L. Carson, "Rapid elastic image registration for 3-D ultrasound," IEEE Trans. Med. Imag., vol. 21, pp. 1384-1394, Nov. 2002.

[12] S. Belongie, J. Malik, and J. Puzicha, "Shape matching and object recognition using shape contexts," IEEE Trans. Pattern Anal. Machine Intell., vol. 24, pp. 509-522, Apr. 2002.

[13] R. Bajcsy and S. Kovacic, "Multi-resolution elastic matching," Comput. Vis., Graph. Image Processing, vol. 46, pp. 1-21, 1989.

[14] C. Broit, "Optimal registration of deformed images," Ph.D. dissertation, Univ. Pennsylvania, Philadelphia, 1981.

[15] B. C. Vemuri, J. Ye, Y. Chen, and C. M. Leonard, "A level-set based approach to image registration," in Proc. IEEE Workshop on Mathematical Methods in Biomedical Image Analysis, 2000, pp. 86-93.

[16] R. Goldenberg, R. Kimmel, E. Rivlin, and M. Rudzsky, "Fast geodesic active contours," IEEE Trans. Image Processing, vol. 10, pp. 1467-1475, Oct. 2001 
[17] C. Davatzikos and J. L. Prince, "Brain image registration based on curve mapping," in Proc. IEEE Workshop on Biomedical Image Analysis, 1994, pp. 245-254.

[18] C. Davatzikos, "Nonlinear registration of brain images using deformable models," Proc. IEEE Workshop Mathematical Methods in Biomedical Image Analysis (MMBIA), pp. 94-103, 1996.

[19] X. Han, C. Xu, and J. L. Prince, "A topology preserving level set method for geometric deformable models," IEEE Trans. Pattern Anal. Machine Intell., vol. 25, pp. 755-768, June 2003.

[20] P. Thevenaz, U. E. Ruttimann, and M. Unser, "A pyramid approach to subpixel registration based on intensity," IEEE Trans. Image Processing, vol. 7, pp. 27-41, Jan. 1998.

[21] D. Shen and C. Davatzikos, "HAMMER: Hierarchical attribute matching mechanism for elastic registration," IEEE Trans. Med. Imag., vol. 21, pp. 1421-1439, Nov. 2002.

[22] H. Lester and S. R. Arridge, "A survey of hierarchical nonlinear medical image registration,” Pattern Recogn., vol. 32, pp. 129-149, 1999.

[23] Y. Amit, "A nonlinear variational problem for image matching," SIAM J. Scientific Comput., vol. 15, pp. 207-224, 1994.

[24] T. R. Downie, L. Shepstone, and B. W. Silverman, "A wavelet based approach to deformable templates," in Proceedings in Image Fusion and Shape Variability Techniques. Leeds, U.K.: Leeds Univ. Press, 1996, pp. $163-169$.

[25] Y. T. Wu, L. F. Chen, P. L. Lee, T. C. Yeh, and J. C. Hsieh, "Discrete signal matching using coarse-to-fine wavelet basis functions," Pattern Recogin., vol. 36, pp. 171-192, 2003.

[26] J. Deubler and J. C. Olivo, "A wavelet based multiresolution method to automatically register images," J. Math. Imag. Vis., vol. 7, pp. 199-209, 1997.

[27] S. Gefen, "Wavelet-based nonlinear multi-dimensional registration," Ph.D. dissertation, Elect. Comput. Eng., Drexel Univ., Philadelphia, PA, 2002.

[28] M. I. Miller, G. E. Christensen, Y. Amit, and U. Grenander, "Mathematical textbook of deformable neuroanatomies," in Proc. Nat. Acad. Sci., vol. 90, 1993, pp. 11944-11948.
[29] J. C. Gee, D. R. Haynor, M. Reivich, and R. Bajcsy, "Finite element approach to warping of brain images," Proc. SPIE (Medical Imaging 1994: Image Processing), vol. 2167, 1994.

[30] T. Rohlfing, C. R. Maurer, D. A. Bluemke, and M. A. Jacobs, "Volumepreserving nonrigid registration of MR breast images using free-form deformation with an incompressibility constraint," IEEE Trans. Med. Imag., vol. 22, pp. 730-741, June 2003.

[31] F. J. P. Richard and L. D. Cohen, "A new image registration technique with free boundary constraints: Application to mammography," Comput. Vis. Image Understanding, vol. 89, pp. 166-196, 2003.

[32] P. J. Besl and N. D. McKay, "A method for registration of 3-D shapes," IEEE Trans. Pattern Anal. Machine Intell., vol. 14, pp. 239-256, Feb. 1992.

[33] M. Vetterli and J. Kovacevic, Wavelets and Sub-band Coding. Englewood Cliffs, NJ: Prentice-Hall, 1995.

[34] G. Strang and T. Nguyen, Wavelets and Filter Banks. Reading, MA: Wellesley-Cambridge, 1997.

[35] S. Mallat, A Wavelet Tour of Signal Processing. New York: Academic, 1999.

[36] I. Daubechies, Ten Lectures on Wavelets. Philadelphia, PA: SIAM (Soc. Ind. Appl. Math), 1997.

[37] J. Nocedal and S. J. Wright, Numerical Optimization. Berlin, Germany: Springer-Verlag, 1999.

[38] D. Kozinska, O. J. Tretiak, J. Nissanov, and C. Ozturk, "Multidimentional alignment using the Euclidean distance transform," Graphical Models and Image Processing, vol. 59, pp. 373-387, 1997.

[39] D. W. Marquardt, "An algorithm for least-squares estimation of nonlinear parameters," J. Soc. Ind. Appl. Math., vol. 11, pp. 431-441, 1963.

[40] C. Ozturk. (1997) Alignment Package for Matlab. [Online]. Available: http://cbis.ece.drexel.edu/ICVC/Align/align.html 